\title{
Resistance to Antimicrobians - A Global Problem with Sectoral Resolution
}

\author{
Sorin Rugină*
}

Ovidius University of Constanța, Romania

Mankind has been and still is constantly threatened by infectious diseases. Antimicrobials, used to treat infections, are considered one of the greatest discoveries of the 20th century because they saved millions of lives from diseases that had a high mortality rate. Mankind has been and is still constantly threatened by infectious diseases. Antimicrobials, used to treat infections, are considered one of the greatest discoveries of the $20^{\text {th }}$ century because they saved millions of lives from diseases that had a high mortality rate. Current infectious pathology is worryingly extending due mainly to "globalization", which confirms the current concept of "Infections Without Borders". In this context, both the consumption of antimicrobial substances and, inherently, the resistance of the main pathogens involved have increased. Unfortunately, antimicrobials have become victims of their success because their abusive use in humans and animals has led to the emergence of resistance among clinically important pathogens. Each dose of antibiotic creates selective evolutionary pressures, resulting in pandemic spread of highly resistant bacterial clones. Resistance to antibiotics is one of the greatest threats to human health. A return to the pre-antibiotic era would not only make possible the development of epidemics caused by multidrug-resistant bacteria, a major threat to the population, but would also jeopardize some of the most valuable therapies in modern medicine, such as transplantation and immunosuppressive chemotherapy programmes -dependent on supportive antimicrobial treatments.

Antibiotics have been abusively used in both humans and animals because of their low cost, uncontrolled availability, poor understanding of their curative limits, and the interference of economic factors (the use of antibiotics as promoters of animal husbandry).[1]

The more antibiotics used (even if this use is medically justified), the more likely the pathogenic bacteria will develop resistance. The situation in Romania, where multi-drug resistant bacteria have become a major threat to human health, is worrying because there are few antibiotics available against such highly resistant bacteria.[2] The World Health Organization (WHO) currently considers antibiotic resistance to be one of the "three major threats to human health" in the coming decades.

Antibiotics, active and effective yet, often have major side effects, or are extremely expensive. More worrying are the occasional reports of resistance to these "last resort" antibiotics (such as carbapenems, tigecycline, linezolid, colistin and daptomycin). The resistance to some of these antibiotics has increased rapidly, selecting multiresistant germs, the most eloquent example being the emergence of multi drug-resistant Klebsiella pneumoniae in Southern European hospitals. [3]

This phenomenon affects not only the pathogenic flora but also the commensal one. Recent researches suggest that the portage of commensal bacteria that have acquired resistance genes is increasing.[4] Even in areas where antibiotic use is non-existent or minimal, up to $50 \%$ of the population is resistant to multi-resistant E. coli.[5] Clearly, antibiotic-resistant bacteria have become so widespread on the planet that they have become a common resident of our body and can easily spread between individuals (for example, from mother to child or within a family).

A slow pandemic spread of resistance clones is developing, and it may be unnoticed if there is no clear and transparent corrective action.

Resistance to carbapenems is particularly worrying because these molecules, initially considered as "reserve" antibiotics, are currently commonly used in hospitals.

Carbapenemases are enzymes that confer resistance to the class of carbapenemic antibiotics. Carbapenemase NDM-1 (an abbreviation of beta-lactamase-1 metal in New Delhi) has recently occurred but has expanded widely, and NDM-1G carbapenemase is al- 
ready disseminated in South Asia's water and sewage systems, spreading subsequently all over the world.

In this extremely complex context, which is the role of doctors? Physicians, both human and veterinary, are those who prescribe antimicrobial treatments. In the absence of coherent policies on the management strategy and control of antibiotic use at national level, as well as of modern and fast microbiological diagnostic tools, their administration is often insufficiently documented by evidence, "empirical" sometimes even "irrational".

Hospitals concentrate the largest number of such multi-resistant germs, with patients and care staff being exposed to varying degrees of risk of getting a healthcare associated infection.[6] These infections are very common (one in eighteen patients in European hospitals are affected by healthcare associated infections [7]) and can be life-threatening, especially when caused by bacteria resistant to different drugs.

Among the most important antibiotic-resistant bacteria in hospitalized patients are the so-called "ESKAPE" pathogens. These are Enterococcus faecium, Staphylococcus aureus, Klebsiella pneumoniae, Acinetobacter baumannii, Pseudomonas aeruginosa and the Enterobacter species [8]. In addition to the ESKAPE pathogens, Escherichia coli (E. coli), which is present in the human intestine, remains the leading cause of mortality related to severe sepsis in hospitalized patients and Clostridium difficile is an important cause of antibiotic-associated diarrhea. They are often resistant to antibiotics and can cause life-threatening complications.

The highest risk of infection is experienced by those admitted into the intensive care services, and especially by critically ill patients. A one-day prevalence study (May 8, 2007) showed that out of 13,796 patients in 1,256 intensive care units (ICUs), in seventy-five of them, $51 \%$ of patients in the ICU were found to be infected. The mortality due to infection of patients assisted in ICU was twice as high as that of patients hospitalized in other sections [8]. It is necessary to approach the critical patient with a multidisciplinary team, which necessarily includes, from the very beginning, a specialist in infectious diseases. His/her participation must be active in the initial assessment of the infectious risk, in the development of an individual plan for the prevention of health care-associated infections, in determining the appropriateness of establishing antimicrobial therapy and in the recommendation and management of anti-infective therapy.[6]
Dramatically, most hospital-acquired infections are of endogenous origin, coming from the patient's own microbiota [9]. An aging population and an increased prevalence of obesity and diabetes mellitus, which are global trends, will cause a significant increase in vulnerable patients, causing the greatest burden in terms of human morbidity and mortality.

The global and multilateral problem of antimicrobial resistance (AMR) necessitates a comprehensive analysis and creative solutions that require action from several sectors of society.

The "One Health" concept recognizes that human and animal health is closely linked to interactions through direct physical contact with farms and animals, the food chain and the environment. High levels of antibiotic-resistant microorganisms and antibiotic resistance genes are excreted in human and animal feces, which contaminate the environment and interact with the soil. Fecal contamination, dust and insects spread microorganisms and resistance genes from humans and animals to the environment [10], with significant ecotoxicological consequences for the microbiosphere.

The economic consequences of these phenomena are enormous. Various studies have attempted to evaluate the morbidity and mortality caused by germs resistant to antimicrobial therapy. ECDC/ EMEA (the European Centre for Disease Prevention and Control/ European Medicines Agency) estimated that, in 2007, resistant strains in Europe caused about 400,000 infections, more than 25,000 additional deaths and 2.5 million additional days of hospitalization.[1]

It is a tremendous responsibility of all those involved to undertake concrete and effective actions in view of establishing the supervision and control of this threatening phenomenon. Research, which provides knowledge, will need to set clear targets for the synthesis of new antimicrobial molecules and alternatives to antibiotics (vaccines). The introduction of new antibiotics will be stimulated by the modernization of diagnostic microbiology, which should be able to quickly identify the causative agents of infections, allowing the predominant use of narrow-spectrum antibiotics. Several antibiotics developed in the past are no longer used due to pharmacokinetics, to pharmacodynamics or to side effects. Research could now "resuscitate" these antibiotics, in order to improve clinical efficacy and to reduce unwanted effects and reuse them as safe and effective antimicrobial drugs in modern clinical practice. Investigations are also needed to optimize consumption, 
dosage and delivery, to improve the antibacterial efficacy of existing antibiotics and to reduce their negative impact on the normal microbiota. In particular, the application of nanomedicine in the delivery of antibiotics (e.g. by coating or encapsulating antibiotics on or in nanoparticles or liposomes) shows great promise.

Programs are needed to implement the monitorization of antibiotic resistance in patients, both in the hospital and in the community, as well as among the healthy population, which include both phenotypic and genotypic analyses, especially of bacterial clones associated with antibiotic resistance genes.

There is a need for concrete action to determine whether food is an important vector for spreading antimicrobial resistance, the role of migration, that of tourism, that of various healthcare systems and the role of agriculture. The growth of global trade and travels are factors, which are certain to favor the spread of antimicrobial resistance between countries and continents.

Knowledge on the exact role of different reservoirs in the environment (e.g. surface water, the soil, the air) in the emergence and diffusion of antimicrobial resistance needs to be deepened, to control and minimize the spread.

Understanding the biological process underlying these phenomena will generate effective preventive measures and countermeasures, provided there is a global approach to the problem, with sector-specific measures.

Clearly, the initiatives of several international organizations addressing antibiotic resistance, such as the World Health Organization, the European Commission, the European Centre for Disease Prevention and Control (ECDC) and ReAct (Action on Antibiotic Resistance) have been very successful in raising the global level of awareness regarding the imminent dangers threatening humanity. Experts in the field must provide innovative approaches to ensure that we can successfully treat the bacterial infections of the $21^{\text {st }}$ century.

\section{- CONFLICT OF INTERESTS}

None to declare

\section{REFERENCES}

1. Joint Programming Initiative on Antimicrobial Resistance Strategic Research Agenda, available at: https://www.jpiamr.eu

2. World Economic Forum (2013). Global Risks 2013. Eighth Edition. - An initiative of the Risk. Response Network. Accessed on the 3rd of April 2013: http://www3.weforum.org/docs/WEF_ GlobalRisks_Report_2013.pdf

3. Tzouvelekis LS, Markogiannakis A, Psichogiou M, Tassios PT, Daikos GL. Carbapenemases in Klebsiella pneumoniae and Other Enterobacteriaceae: an Evolving Crisis of Global Dimensions. Clin Microbiol Rev. 2012;25:682-707.

4. Forslund K, Sunagawa S, Kultima JR, et al. Country-specific antibiotic use practices impact the human gut resistome. Genome Res. 2013;23:1163-69.

5. Pallecchi L, Bartoloni A, Riccobono E, et al. Quinolone Resistance in Absence of Selective Pressure: The Experience of a Very Remote Community in the Amazon Forest. PLoS Negl Trop Dis. 2012:6: 1790. doi:10.1371/journal.pntd.0001790

6. Rugina S, Dumitru IM. The Role of infectious diseases specialist in critical patient assistance, J Crit Care Med. 2018;2(Suppl 1):24.

7. ECDC Surveillance Report. Point prevalence survey of healthcare-associated infections and antimicrobial use in European acute care hospitals 2011-2012. Accessed on the 11th of April 2013: http://www.ecdc.europa.eu/en/publications/ Publications/Forms/ECDC_DispForm.aspx?ID=1155

8. Rice LB. (2008).- Federal funding for the study of antimicrobial resistance in nosocomial pathogens: no ESKAPE. J Infect Dis. 2008;198:1079-81.

9. Grundmann H, Bärwolff S, Tami A, et al. How many infections are caused by patient-to-patient transmission in intensive care units? Crit Care Med, 2005;33:946-51.

10. Silbergeld EK, Graham J, Price LB. Industrial Food Animal Production, Antimicrobial Resistance, and Human Health. Annu Rev Public Health. 2008;29:151-69. 